\title{
THE CONDUCTION VELOCITY IN RELATION TO THE STIMULATION INTENSITY AND TO TḦE SIZE OF THE ACTIVATED AREA (OBSERVATIONS ON LILLIE'S NERVE MODEL)
}

\author{
By KazUmi YAMAGIWA \\ Department of Physiolozy, Tokyo Medico-Dental College, Tokyo
}

\section{INTRODUCTION}

The relation between the conduction velocity and the stimulation intensity has often been a subject of investigation because of its fundamental importance in nerve physiology. After a period of indetermination, the general opinion seemed to have once settled that the velocity is invariable, not depending on the stimulation intensity (Engelmann (1), Nicolai (2)): Recently, however, we have again a few reports which contradict to the above conclusion. According to Iizumi (3), the conduction velocity of the leaf-stalk of Mimosa pudica varies greatly with the intensity of the stimulation. Shibaoka (4) showed on the same material a clear increment and decrement of the velocity in the vicinity of the stimulated point. Gelfan (5) found on verve that the distance travelled and also the conduction velocity get larger with larger stimulation intensity or with larger diameter of the stimulating electrode. On the other hand, the increment and decrement, also the increase and decrease, of the velocity, are all possible theoretically, if only the size of the area primarily excited can be made large or small properly (Yamagiwa $(6,7)$ ). We should notice here that in usual methods of stimulation, the variation of the intensity brings forth necessarily that of the area stimulated. So, to answer the question whether the velocity varies with the stimulation intensity or not, we have first to invent a method in which we can control the intensity or the area only, and then to examine carefully whether the velocity varies or not 1 ) with the intensity when a certain definite area is stimulated, 2) with the size of the stimulated area and 3) with distance from the stimulating electrode. It has long been one of the author's desires to perform these examinations 'on the nerve itself, but owing to the methodical difficulty they have not been realized yet. That is why he took up Lillie's model in the present work. It turned out after the experiments, however, that an exact method cannot be established even in the model, and, consequently, we cannot get any definite answer for the question. Still it was not without results, about which we should like to describe here briefly. As to the methodical details, the reader is referred to the previous paper (Yamagiwa (7)). Here, just a few particular points will be described.

I. The conduction velocity in relation to the stimulation intensity

1. The first method adopted and the results obtained (Fig. $1 \& 2$ ). 


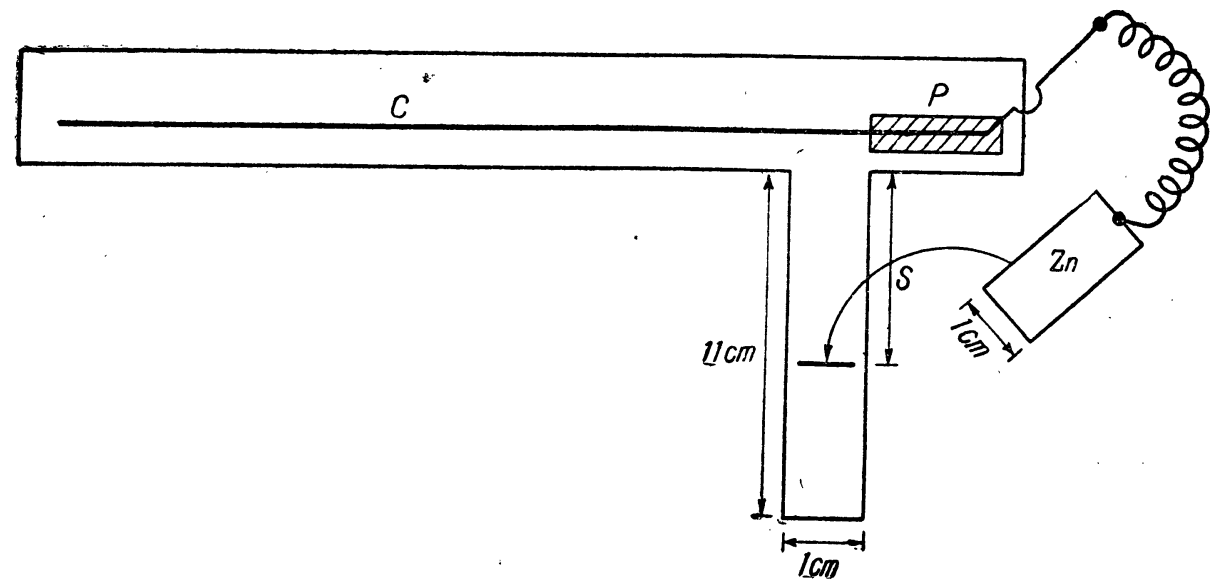

Fig. 1.

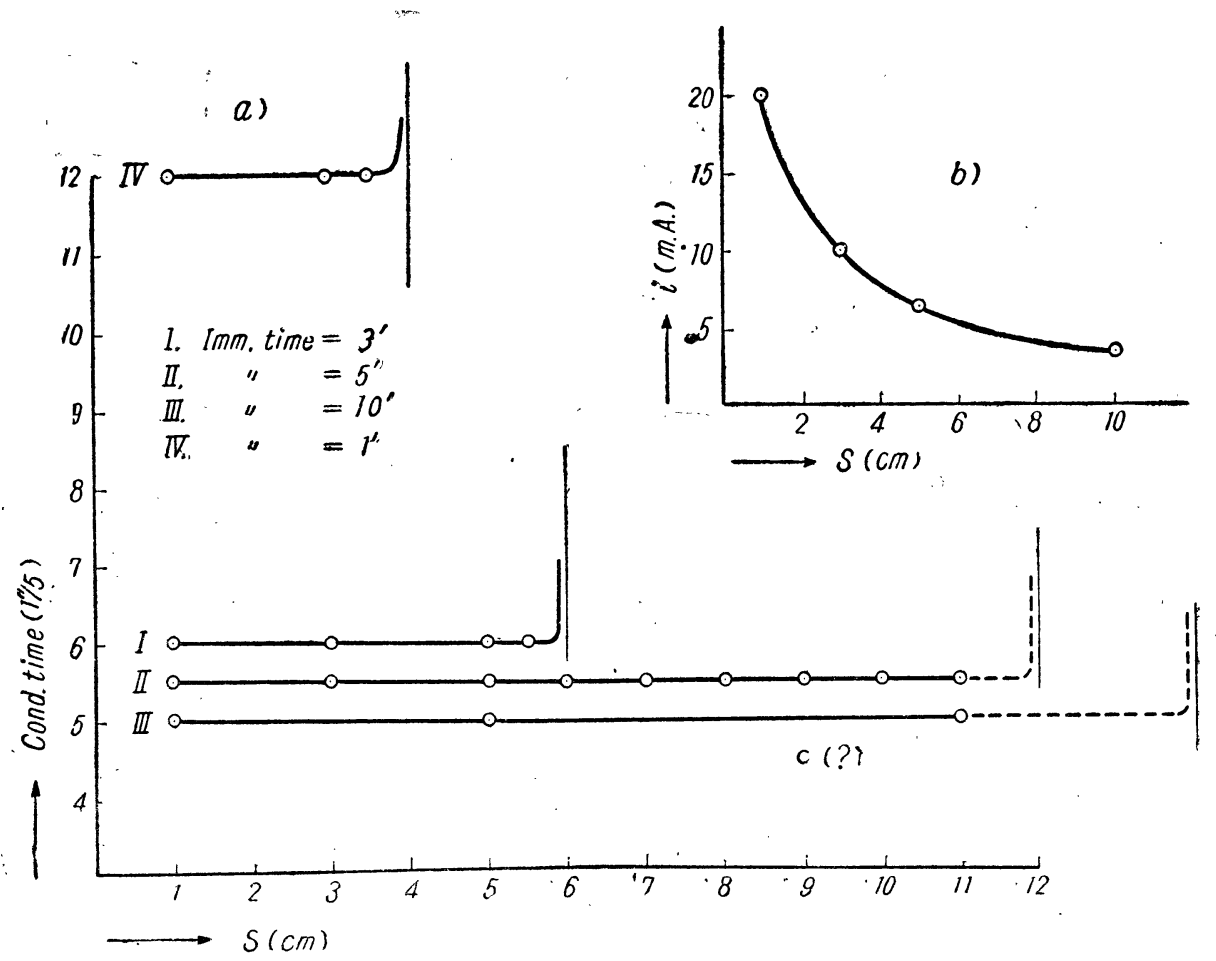

Fig. 2.

The container for the model is an asymmetrical $\mathrm{T}$-shaped groove, a short sidegroove being attached near to one' end of the long principal groove in right angles. It was filled with strong nitric acid (specific gravity $=1.35$ ) to hold the core in it. The core was $30 \sim 40 \mathrm{~cm}$ in length, of which some parts at the right end was covered with: paraffin ( $P$ in Fig. 1). It was placed along the principal groove and as near as the side groove, and that in such a position that the left edge of the P-part came in line with the right wall of the side groove. The stimulus was given by a sudden inser- 
tion of a blade of zinc metal to a certain measured place in the side groove. An. electrical current will then flow out of the zinc into the passive core to activate it. Thereby the lines of current will run almost parallel to the walls of the side groove, because the zinc blade was made just about so wide as the breadth of the side groove, and, on the other hand, the internal resistance of the core can be taken as. zero. Consequently, the part of the core exposed to this current (that is, about $1 \mathrm{~cm}$ in this case) will be activated almost uniformly, the intensity of the stimulation being controlled by putting the zinc electrode at various distances from the core. (If we have a nerve in place of the core, the most of the stimulating current will flow into it at the right edge because of the large internal resistance, and we shall rather have a "point-stimulation" instead of an "area-stimulation". In the case of the model, on the contrary, we should rather think of some enlargement of the area tobe stimulated, because there will be more or less "current spread" owing to the: polarisation taking place at the boundary between the metal and the fluid, to which we shall come back again).

The measurements were made on the conduction times of the activation waves thus provoked, by means of a stop-watch of $1^{\prime \prime}{ }_{5}$ unit. All the results ob tained showed beautifully that the conduction time is quite independent from the stimulation: intensity. An example of series of measurements is shown in Fig. 2, a). The bystanding figure (Fig. $2, b$ ) is showing the $\mathrm{i}$ (m.A)-S (the distance between the core and the zinc electrode)-relation examined by a milliammeter directly after activations. It can't be very exact, but is enough to show how the current varied with $S$.

The vertical lines standing on one side of each curve are showing the critical. Ss, where the stimulation is just ineffective, or, in other words, the conduction time appears infinity. We learnt, thus, by this method firstly that the conduction velocity is independent from the stimulation intensity, and secondly, that the conduction velocity gets smaller, while the threshold gets higher, with shorter immersion times, that is, with shorter stimulation intervals. These are facts just corresponding to those in nerve taking place in the relative refractory period. Whether the supernormal phase exists or not in the model, was not pursued.

2. The second method adopted and the results obtained (Fig. $3 \sim 4$ ).

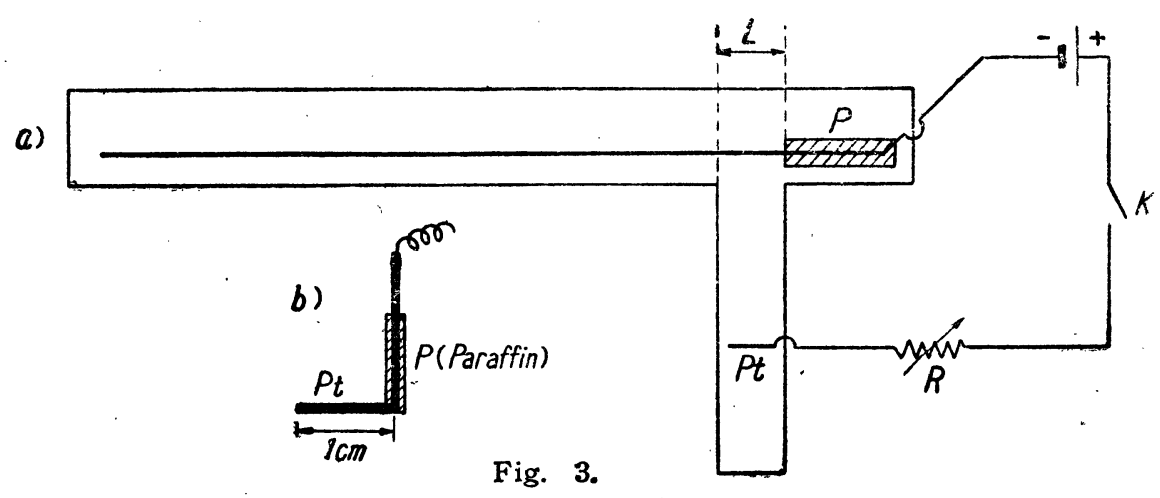


The whole apparatus used in the first method was employed in this case, too. The only difference was that an ordinary electrical circuit was used for stimulation, replacing the zinc electrode with a platinum one shaped as shown in Fig. $3, b$ ). In practice, the position of the electrode was fixed at a certain distance (about $2 \mathrm{~cm}$ from the core), and the intensity of the stimulating current was controlled by means of the source battery and a variable resistance. The stimulation was made to start with the closure of the knock-down key $(K)$ and to finish, by the break of it, at the very moment when the activation wave appeared. Fig. 4 is an illustration of the results obtained, which quite agrees with those obtained by the first method.

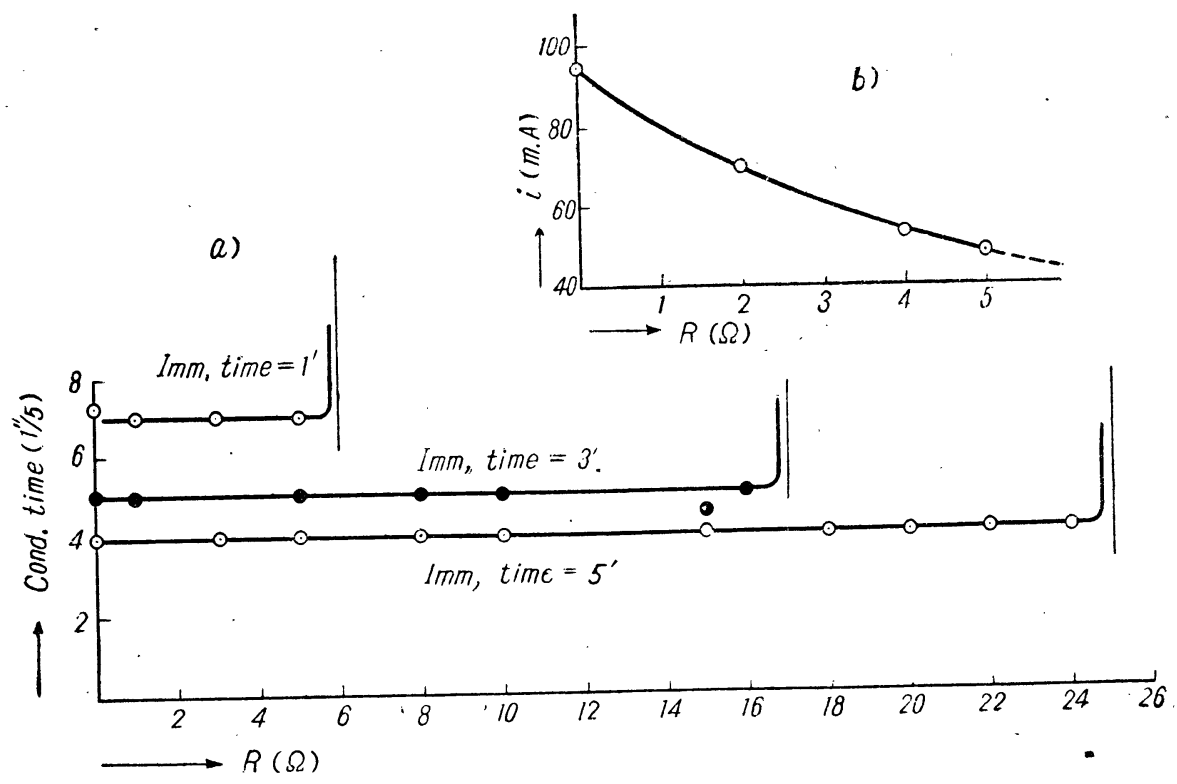

- Fig. 4.

II. The conduction velocity in relation to the area activated

1. The first method adopted and the results obtained (Fig. 5 6).

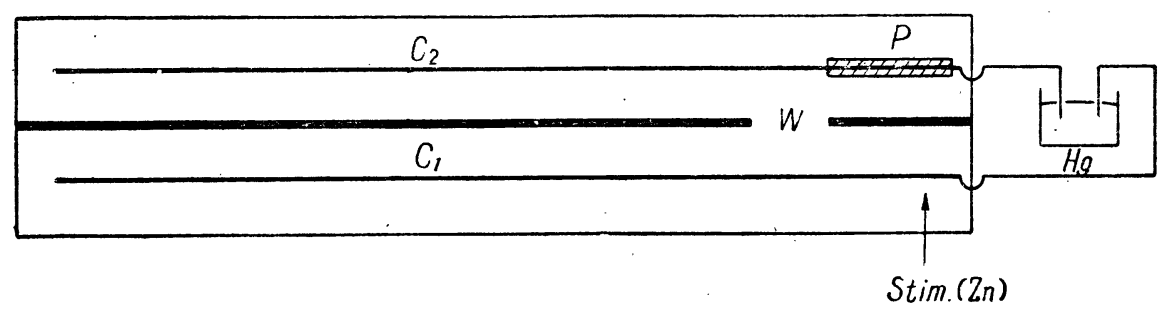

Fig. 5 .

In Fig. 5, $C_{1,2}$ represent two iron cores made of one and the same material. They are connected by means of $\mathrm{Hg}$ outside the container. $W$ is a small window cut out of the separating wall, through which the fluids on both sides communicate with each other. $P$ means paraffin for covering some end-part of the core $C_{2}$. 
After a sufficient passivation, the core $C_{1}$ was stimulated at the right end by piece of zinc metal. The activation wave proceeds then towards the left, and when it passes along the window $W$, it provokes an activation wave in the core $C_{2}$ by the activation current flowing in the direction $C_{1} \rightarrow C_{2} \rightarrow H g \rightarrow C_{1}$. Thereby, not only the intensity of the stimulating current, but also the area to be activated, will vary with the size of $W$, and, therefore, if the conduction velocity in $C_{2}$ is found to vary with the size of $W$, we can conclude that the velocity depends on the area primarily activated, because we learnt in the previous section that the stimulation intensity has no influence on the conduction velocity.

Actually, now, the velocity of the conduction in $C_{2}$ did vary with $W$. It was found: the smaller, the smaller the window was (Fig. 6). This tendency was quite clear. in the cases of short immersion times, less clear in longer immersion times (Fig.6, II \& III), and finally almost disappearing in a very long immersion time, as though the velocity was independent from the size of $W$ (Fig. 6. I.). There are, generally, liminal breadths of $W$, which are just necessary for starting the waves in $C_{2}$ in each immersion time. They are shown by the positions of the vertical lines drawn by the side of each curve. It is noteworthy that they get smaller and smaller with larger and larger immersion times.

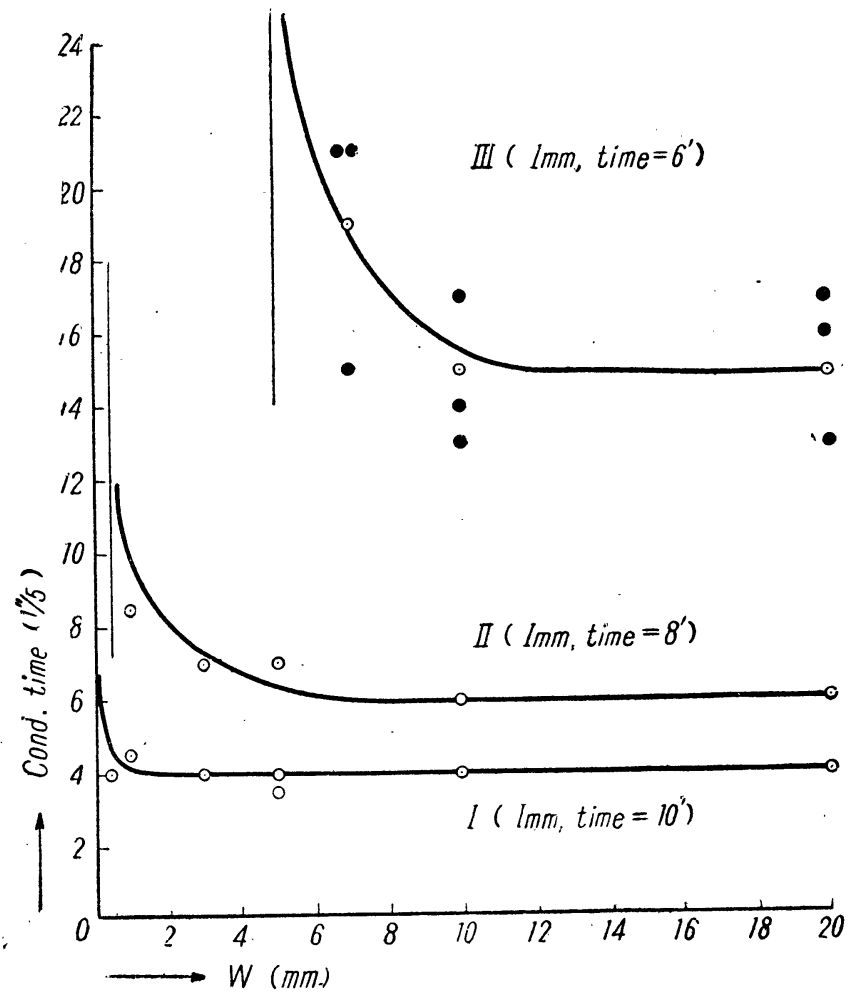

Fig. 6.
N. B. 1. The expressions "long" or "short" as to the immersion time bear here just a reliative meaning, the absolute values being widely different from core to core.

N. B. 2. In one half of the experiments, the immersion time was varied for both $C_{1}$ and $C_{2}$, and in the other half, just for $C_{2}$, keeping that of $C_{1}$ constant. But the results were just the same. 
2. The second method adopted and the results obtained (refer to Fig. 3).

a) In the arrangement represented by Fig. 3, the core was moved along in various grades towards the left so as the area $L$, which is directly exposed to the stimulating current, would be varied. The stimulating electrode was fixed at a $2 \mathrm{~cm}$ distance from the core. $L$ was made as large as $10 \mathrm{~mm}$, to begin with, and the resistance $R$ was controlled properly to bring the current intensity just over the threshold. Then, $L$ was made smaller in various grades, to examine how the conduction velocity varies with it.

The first comparison was made between the cases $L=10 \mathrm{~mm}$ and $L=1 \mathrm{~mm}$, but, contrary to our expectation, we could not find any difference between the conduction times. Fig. 7, I\& II are examples of those. In those cases, the core was not in a constant condition, and did not always give constant results for repeated measurements of one and the same kind, so the measurements were performed on the cases $L=10 \mathrm{~mm}$ and $L=1 \mathrm{~mm}$ alternatively and repeatedly in regular time intervals, and the two curves thus obtained were compared with each other (see N. B. below). As seen in the figure, they run mingled on $\mathbf{a}$ similar course, and one curve cannot be distinguished from the other, which

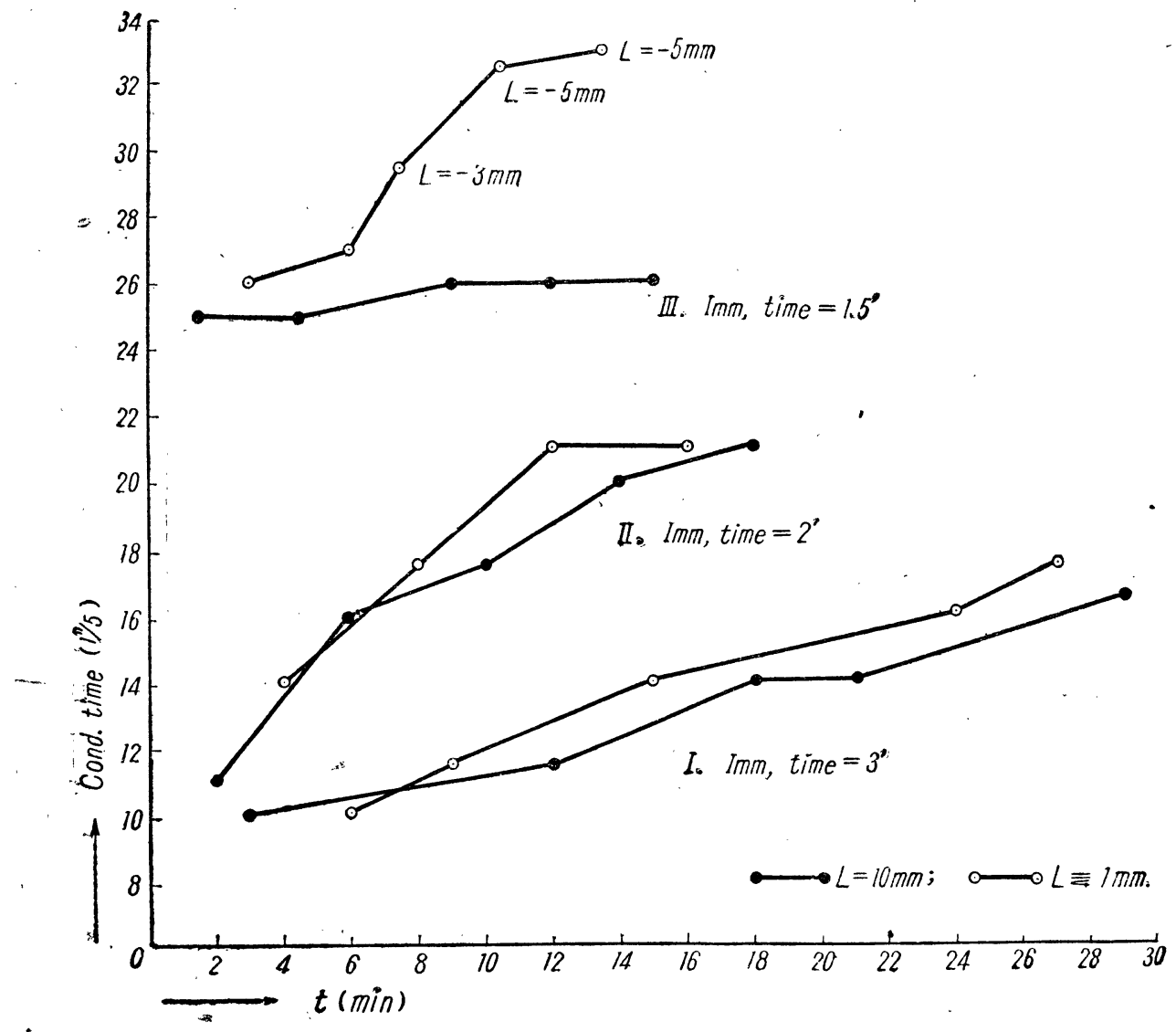

Fig. 7. 
means that the conduction velocity is indifferent, as far as examined, to the area primarily activated. Is it, however, truely so? And always so? Bearing this question in mind, we measured, for trial, the threshold currents necessary for starting the waves in the cases $L=10 \mathrm{~mm}$ and $L=1 \mathrm{~mm}$, and found, again unexpectedly, that the threshold current in the case of $L=1 \mathrm{~mm}$ was so large as $80 \%$ of that in the case of $L=10 \mathrm{~mm}$. It had been assumed that the most of the current would flow into the core almost uniformly at the part $L$, and if this assumption is right, the threshold current in the case $L=1 \mathrm{~mm}$ should be about $10 \%$ of that in the case $L=10 \mathrm{~mm}$. Of course we should take into consideration some current spread due to the polarisation taking place at the phase boundary, but still " $80 \%$ " is too large a value in order that the assumption can be allowed. Perhaps we should take it as showing that the current does spread to a fairly large extent, or else, though not very probable, that the current flows into the core not uniformly but through a narrow region near the extremity. Anyhow, we learnt that it is impossible to activate a certain definite area wanted, even in the model, and in this sense, the present work was unsuccessiul.

But we went on further, instead of giving up the work, and planned to measure the conduction time in the condition $L<0$, because the spreading current is to diminish exponentially with distance and, consequently, the area to be activated is to get smaller as we move the core to the left. Being suggested, further, by the experimental result obtained by the first rethod, we made the immersion time much shorter. The result obtained in these new conditions was such as shown in Fig. 7, 11 , where the test curve $(L<0)$ deviates unmistakiably from the standard curve $(L=10 \mathrm{~mm})$ and makes a nice contrast to Fig. $7,1 \& 11$. This result is showing certainly, although not directly, that the velocity is a function of the area primarily actitated, at least in the cases of short immersion times. This fact was reascertained many times thereafter.

b) Keeping $L=0$, the resistance $R$ was controlled to grade the intensity of the stimulating current, expecting to get the same result as was obtained in a). The results were actually just as they were expected. An example is shown in Fig. 8, where the curve obtained with $R=302$ is taken as the standard.

N. B. Why the stendard curves in Fig. $7 \sim 8$ do not run parallel to the horizontal axis, may be perhaps owing partly to the nature of the core, but chiefly to the "short" immersion time. It has often been experienced in various cases that the conduction velocity, the distance travelled etc. changed with time irregularly or gradually, when measurements of one and the same kind were repeated in short immersion times. 


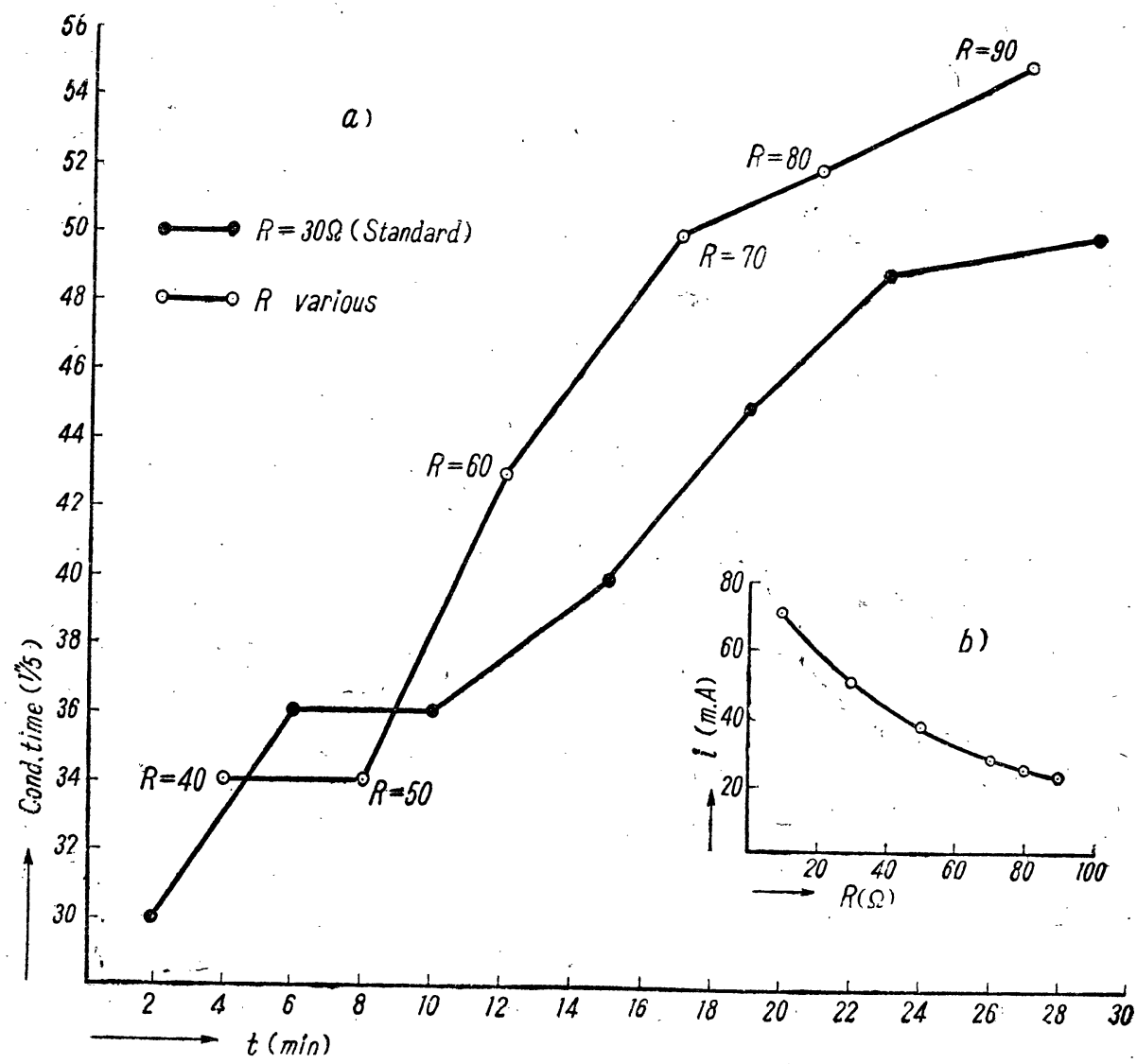

Fig. 8.

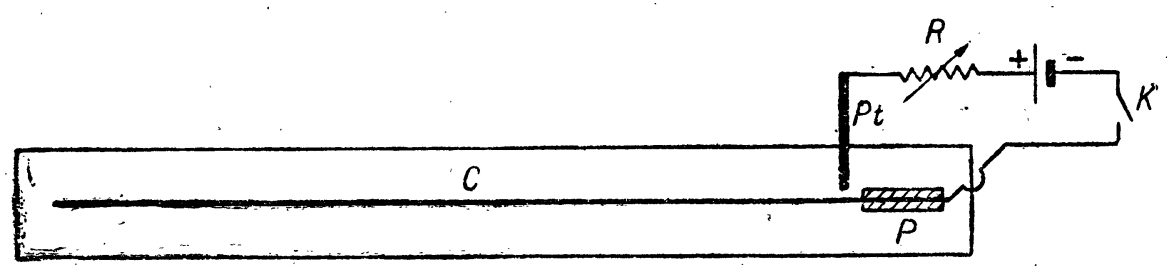

Fig. 9.

3. The third method and the results (Fig. 9).

The most usual way of setting of the stimulating electrode and the most usual electrical circuit were adopted here, because it seemed to be hopeful to do so from the experiences in the first and second methods, deseribed above.

The conduction velocities examined in this way were, however, contrary to our expectation, quite constant in all stimulation intensities tried. The reason is not quite clear, but we think it is very probable that the current spreads much more easily in such an arrangement, and so the area to be stimulated cannot be localized to less than a certain limit. 
4. The fourth method and the results (Fig. 10).

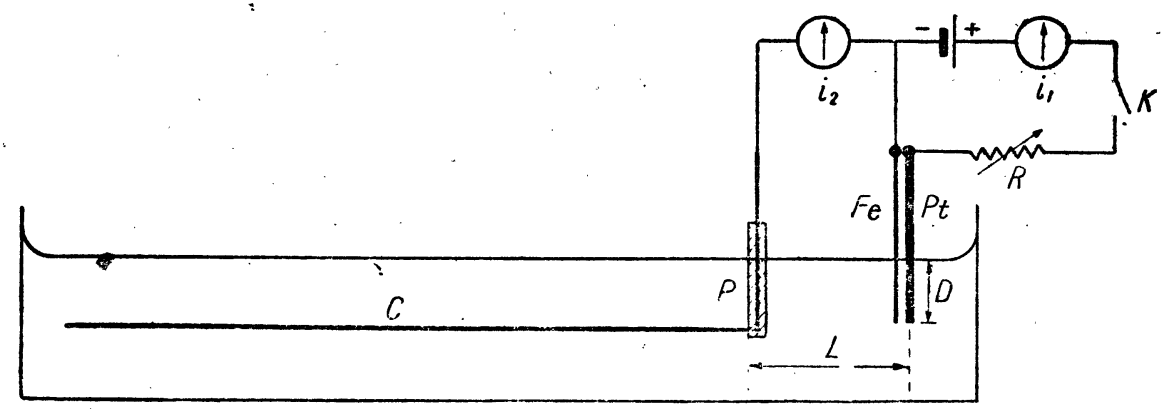

Fig. 10.

In Fig. 10, $P t$ is a platinum electrode and $F e$ is a short iron wire cut from the same sample as the core. The upper half of them is held in a thin glass tube filled up with paraffin, and a part of the exposed lower half $(D$ in the figure) is dipped in the acid bath together with the core, $C$. It was our plan to close the key, $K$, at a proper moment, to activate $F e$ directly, and then to have the core activated by the activation current flowing from $\mathrm{Fe}$ to the core. We were going in this way to examine the conduction valocity for several values of $D$ chosen at random.

The distance separating $P t$ from $F e$ was made as small as possible (perhaps $0.5 \mathrm{~mm}$ or so), while the distance between $P t$ and $C$ was chosen so large as $2 \sim 3 \mathrm{~cm}$, in the hope that the stimulating current would mostiy flow from $P t$ to $F e$ and not from $P t$ to $C$ directly, and thus we could perhaps exclude the direct effect of the so-called current spread. When, however, we examined the currents $i_{1}$ and $i_{2}$ by means of milliammeters, we found that the current $i_{2}$ increased gradually from zero to attain soon a fiairly high vialue, and then, after a sudden increase to a much higher value, to fall to zero immediately. The second variation, that is, the sudden rise and fall, is due to the activation and repassivation of $F e$, but the initial rise is due to the spread of the stimulating current, the rate and grade depending on the length of $D$. Hence, even if the conduction velocity depends really and truely on the length of $D$ (that is, on the area of the primary source), it will be made obscure by the effect of $i_{2}$. For this reason, this method was abandoned.

5. The fifth method and the result (Fig. 11).

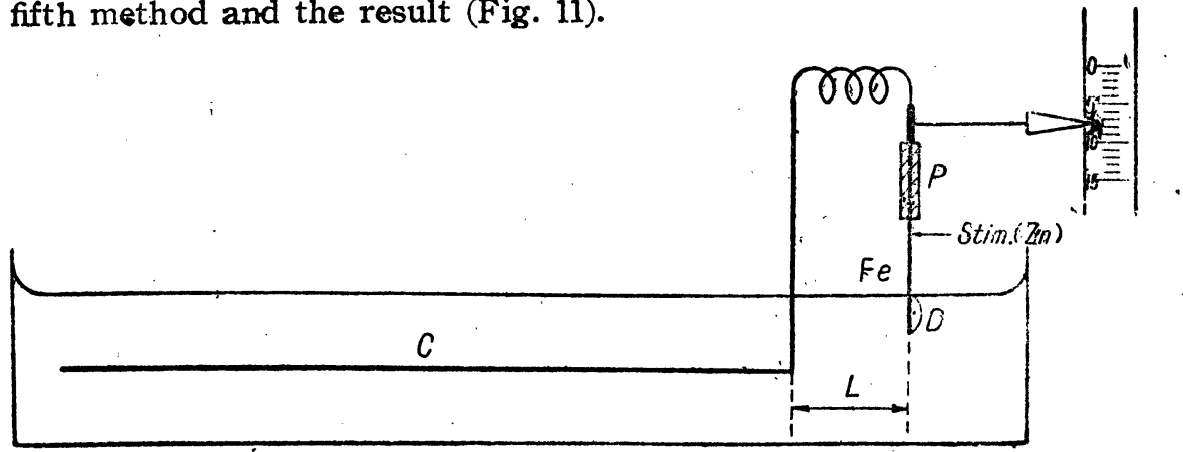

Fig. 11. 
In Fig. 11, $\mathrm{Fe}$ is a short iron wire contiected with the core, $\mathrm{C}$, in the alr. The upper part of $\mathrm{Fe}$ is covered with paraffin, leaving the lower part exposed by some $2 \mathrm{~cm}$. Fe can be moved up- and downwards by a suitable apparatus. To begin with, the whole exposed part of $F e$ is held in the acid to make it passive togather with the core. When we want to stimulate it, we move it upwards, leaving some lowest part $(D)$ in the acid, and touch the uppermost part with a piece of zinc metal. As $F e$ is still wet with nitric acid, an activation wave is evoked, but as the acid exists only in the state of a thin film. the conduction is extremely slow until it reaches the fluid surface, where the velocity changes suddenly into a very rapid one and makes the whole $D$ activated almost, though not exactly, in a moment. The part $D$ thus activated will then activate the core, $C$, so that we can pursue in this way the relation between the conduction velocity and the length of $D$, without being disturbed by the stimulating current itself.

But this method, too, turned out to be unuseful for the object, because it was soon found that there is a threshold in the length of $D$, say $D_{\text {min }}$, which is just necessary and sufficient for starting an activation wave. Suppose, now, the case $D>D_{\text {ninin }}$. The activation wave in $D$ spreads with time, although very rapidly, and there will be a certain moment at which the wave will just occupy the length $D_{\min }$, and so make a wave start in the core. The activation of the remaining part of $D$ will be too late to give influences on the velocity, so it will be non-sense to examine the velocities for various $D s$, because the velocity can never be other than that for $D_{\text {min }}$ itself. We found actually that the velocity was constant, being quite independent from the length of $D$.

Thus, the method which seemed to be very promising, did not meet the present purpose. But it was a big unexpected by-product that it was confirmed experimentall $y$ that there exists a certain $D_{\min }$, which is large or small according to the immersion time short or long. The critical breadth of the window experienced before (see Fig. 6) is perhaps the same thing as $D_{\min }$ manifested in another form, and we think they are both expressing that "a certiain area activated is necessary for the initiation of propagated wave", a conclusion theoretically derived recently by some workers (Rushton (8), Yamagiwa $(6,7)$ ).

\section{Discussions}

1. From the former half of the experiments, a conclusion was derived that the velocity is indifferent to the stimulation intensity, while from the latter halt, a conclusion that the velocity depends on the "area. activated".

The latter conclusion, however, was derived, though not wholly, from the assumption that the area varies with the stimulation intensity, or with the position of the core. But most of what we did actually, was ths measurement of velocities for several stimulation intensities, and if we take the results obtained as they are, some of them are indicating that the velocity depends on the stimulation intensity, 
a fact contrary to the former conclusion.

In the former helf of the experiments, however, a stimulating electrode of an about $1 \mathrm{~cm}$ breadth Wis laid in the side groove of about the same breadth. 'As it was mentioned beiore, the lines of current will be parallel to the side wialls of the groove, and the part of the core directly exposed to this current (that is, about $1 \mathrm{~cm}$ ) will be activated altogether. What we have to take into consideration is the current spread beyond that region and the activation of some extra-part due to it. This extra-part will naturally be large or small according to the current intensity, hence, to vary the current intensity in the arrangement means to activate $1 \mathrm{~cm}$ of the core with various intensilies, on one side, and to vary the magnitude of the extra-part to be activated, on the other. In other words, the length activated can be more or less larger but never smaller than $1 \mathrm{~cm}$. Now, the velocity a.ctually obtained in such a condition was always found to be indifferent to the stimulation intensity. This result means, as far as the above consideration is right, that the velocity depends neither on the stimulation intensity nor on the area activated, if . the area is larger than a certain value.

In the latter half, on the contrary, the area must have been graded finely in a small range near zero, by choosing proper intensities of the current or proper positions of the core. In this point, the circumstance is quite different from that in the former half, and we think that the contradicting result obtained in the latter half, is only apparent and is expressing, in the essense, that the velocity varies with the area activated to a certain extent.

2. Some would say that the shortening of the conduction time in the case. of strong stimulation is not due to a larger velocity but to some dislocation of the starting point to the periphery, owing to a wider current spread. The representztive of this concept is found in the well-known book of $\mathrm{K}$. Lucas ("The conduction of the nervous impulse", 1917), in which he says: "- - If now a stronger current is used, the part of the cathodic region within which the current is dense enough to excite, will be more extended. Over the whole of this region the condition necessary for starting a nervous impulse will presumably be realized. But it will be only from the peripheral part of this region that a nervous impulse will be conducted away, since any impulse which attempis to follow it from the inner part of the excited region will be blocked by the refractory period which the impulse of peripheral origin leaves behind. " (P. 13 14). But, wes he right in saying so ? Is it possible that an impulse starts from a peripherel point, leaving behind a refractory period against the impulise coming down from the inner part? We don't think so, because the current is stronger in the inner part and therefore any point therein is to be excited earlier than the peripheral point adjacent to it. In other words, the impulse starts always from under the electrode, where the current is most elense, to be conducted away to the periphery. The point is that the "conduction" in the cathodic region just mentioned, is not a conduction in the ordinary sense, because any point in the 
rigion receives stimulating currents from two sources, that is, from the battery and from the inner points excited previous to that. The result will be that the excitation at any point will take place much earliex than in the ordinary conduction, and owing to this, the "conduction" in the region will appear to be extraordinarily quick. This is practically equivalent to exciting "a certain length of nerve" in a certain very short time, and this "certain length of nerve" is just what will be varied by the stimulation intensity. The essential question is that this given "length" of nerve excited plays a role or not in determining the conduction velocity in each case.

In the present work on the model, the current was made to flow at the moment of closure of the key, $K$, and to finish at the very moment when the wive was seen to start, by breaking the key. There elapsed inevitably a certain definite time (the reaction time) in breaking the key, during which the wave proceeded a little. This could not be measured out, but was surely just a few $\mathrm{mm}$ at the largest and was variable according to the current intensity. It was equivalent to giving activated area of several dimensions (less than a few $\mathrm{mm}$ in length) as the origin:al source of energy for the conduction.

The conduction time actually measured in this way was found to be shorter, as described before, in the case of stronger stimulation. The difference was not very large, but never so small, either, as to be explained by a few $\mathrm{mm}$ dislocation of the starting point. The very cause of this difference is, we interprete, the larger conduction velocity due to the larger activated area given.

\section{SUMMARY}

The conduction velocity in relation to the stimulation intensity and to the primarily activated area was pursued on Lillie's nerve models. The results obtained will be summarized as follows :

1. The threshold intensity gets smaller and the conduction velocity gets larger, with larger immersion time.

2. The velocity is indifferent to the stimulation intensity in both long and short immersion times.

3. The velocity seems to get larger, to a certain extent, with larger area activated, but, so far, only in the case of short immersion time.

4. For the initiation of conduction, a certain liminal area is needed to be activated as the original source. This area gets smaller with longer immersion time.

5. It was pointed out that an idea widely accepted concerning the effect of the so-called current spread upon the conduction time is mistaken, and a new interpretation was proposed for that.

\section{REFERENCES}

1) Engelmann, Th. W.: Ueber den Einfluss der Reizs.ärke auf die Fortpflanzungsgeschwindigkeit 
der Erregung im quergestreiften Froschmuskel, Pflüger's Archiv, 66, 574, 1897.

2) Nikolai, G. F.: Ueber die Leitungsgeschwindigkeit im Riechnerven des Hechtes. Pflüger's Archiv, 85, 65, 1901.

3) Iiizumi : Stimulation of Mimosa pudica III. Collected papers of the Physiol. Lab. of Jikeikai Medical College, 3, 373, 1943 (in Japanese).

4) Shibaoka, T.: The velocity of excitation conduction in relation to the stimulation intensity. in Mimosa pudica. Kagaku, 18, 560, 1948 (in Japanese).

5) Gelfan, S.: Studies of single muscle fibers. Am. J. Physol., 93, 1, 1930; 95, 412,1930 (with Gerard); 96, 16, 1931.

Action potentials from single muscle fibers. Am. J. Physiol., 101, 678, 1932 (with Bishop). Conducted contractures without action potentials in single muscle fibers. Am. J. Physiol., 103, 237, 1933 (with Bishop).

6) Yamagiwa, K.: The action current as a stimulus. J. Jap. Physiol. Soc., 5, 1, 1940.

7) Yamagiwa, K.: The active area in course of excitation conduction. Jap. Med. J., 1, $339,1948$.

8) Rushton, W. A. H.: Initiation of the propagated disturbance. Proc. Roy*. Soc., B 124, 210, 1937.

The expenses for this work were defrayed by a grant from the National Research Council of Japan. 